\title{
(e,2e) measurements on xenon: Reexamination of the fine-structure effect
}

\author{
R. Panajotovic, J. Lower, and E. Weigold \\ Atomic and Molecular Physics Laboratories, RSPHYSSE, The Australian National University, Canberra, ACT, Australia 0200
}

A. Prideaux and D. H. Madison

University of Missouri, 1870 Miner Circle, Rolla, Missouri 65409, USA

(Received 25 October 2005; revised manuscript received 27 January 2006; published 1 May 2006)

\begin{abstract}
The process of electron scattering from heavy target atoms is of considerable interest due to the enhanced role of relativistic effects and distortion of the electron trajectories resulting from the large value of nuclear charge. Here we present (e,2e) ionization measurements and distorted-wave Born approximation calculations for the scattering of spin-polarized electrons from xenon atoms in which the fine-structure levels of the residual ion are resolved. Comparison of measurements performed using a high-sensitivity toroidal analyzer spectrometer with the predictions of sophisticated calculations provide an improved understanding of the ionization dynamics of heavy target atoms and the treatment of electron exchange processes.
\end{abstract}

DOI: 10.1103/PhysRevA.73.052701

PACS number(s): 34.80.Dp, 34.80.Nz

\section{INTRODUCTION}

\section{A. Background}

Investigations into the impact-induced ionization of atomic targets are of considerable importance as they provide detailed insight into the many-body behavior of systems of charged particles mediated through the Coulomb interaction, the basis for the electronic structure of matter. In particular, a detailed understanding of mechanisms that underlie this process is crucial to our understanding of a variety of phenomena, many of technological importance. Examples are the physics and chemistry of the upper atmosphere, gas discharges, fusion physics and the operation of lasers.

The most detailed information on single ionization dynamics comes from measurements performed within a kinematically complete experimental framework. This is achieved in the present case through the application of (e,2e) electron coincidence techniques in which pairs of electrons derived from a common collision event are identified by their relative arrival times at two separate detectors [1]. Measurement of the momenta of the two final-state continuum electrons $\boldsymbol{p}_{\mathbf{1}}$ (energy $\boldsymbol{E}_{\mathbf{1}}$ ) and $\boldsymbol{p}_{\mathbf{2}}$ (energy $\boldsymbol{E}_{\mathbf{2}}$ ) and knowledge of the primary electron momentum $\boldsymbol{p}_{\boldsymbol{i}}$ (energy $\boldsymbol{E}_{\boldsymbol{i}}$ ) enables the recoil momentum of the ion $\mathbf{q}$ and the binding energy $\varepsilon$ of the electron removed from the target in the ionization process to be determined from energy and momentum conservation:

$$
\begin{gathered}
q=p_{i}-p_{1}-p_{2}, \\
\varepsilon=E_{i}-E_{1}-E_{2} .
\end{gathered}
$$

The present work involves the electron impact ionization of ground-state xenon atoms by spin polarized electrons in which the $5 p^{5}{ }^{2} P_{1 / 2}$ and ${ }^{2} P_{3 / 2}$ fine-structure levels of the residual ion, separated by $1.3 \mathrm{eV}$, are energetically resolved. In detail, the reaction considered is

$$
\begin{aligned}
e^{-}\left(E_{i}\right)(\uparrow \downarrow)+\operatorname{Xe} 5 p^{6}\left({ }^{1} S_{0}\right) \rightarrow & \mathrm{Xe}^{+} 5 p^{5}\left({ }^{2} P_{1 / 2},{ }^{2} P_{3 / 2}\right) \\
& +e^{-}\left(E_{1}\right)+e^{-}\left(E_{2}\right),
\end{aligned}
$$

where $\uparrow$ or $\downarrow$ represent either spin up $\left(m_{s}=+1 / 2\right)$ or spin down $\left(m_{s}=-1 / 2\right)$ for the electron initiating the ionizing collision. The purpose of the measurements is to sensitively probe the nature of the electron exchange effects in the process of electron impact ionization and to test the accuracy of state-of-the-art scattering calculations. The reasons for the present choice of target and reaction kinematics are given below.

\section{B. Spin resolved (e,2e) ionization measurements}

Over recent years improvements in experimental techniques have enabled highly quantum-state-specific ionization experiments to be performed through the application of a variety of advanced approaches. These include the employment of beams of spin-polarized electrons [2,3] and/or beams of spin-polarized target-atoms [4] and the laser preparation of atoms in specific angular momentum projection states corresponding to oriented or aligned atomic orbitals [5]. As a result of these improvements, enhanced quantum-state specificity in measurements has been achieved, enabling selective aspects of many-body electron behavior to be isolated and theoretically modeled. These include the role of orbital angular momentum transfer between projectile and target electrons, contributions from direct and exchange scattering processes, details on the coupling between orbital and spin angular momentum, and the influence of relativistic effects in scattering and in determining atomic structure.

For light atomic targets and low electron impact energies, the effects of explicit spin-dependent forces acting on the initial and final-state continuum electrons is negligible and nonrelativistic scattering theory can be used to describe the experimental results. Baum et al. [4,6,7] performed (e,2e) measurements on the ionization of light spin-polarized lithium atoms with polarized electrons at an electron impact energy of $54.4 \mathrm{eV}$. They observed strong spin asymmetries 
which were explicable by the nondistinguishability of the two scattered electrons in the final state (electron exchange effects). These measurements provided stringent tests to scattering theory and enabled the cross section ratio for singlet to triplet scattering to be deduced.

For experiments at high impact energies and involving heavy target atoms, spin-flip processes will occur and other relativistic effects come to prominence. Under such circumstances, a fully relativistic treatment of the scattering process is required to accurately describe the experimental data. Using spin-polarized electrons and high impact energies, Nakel and collaborators $[8,9,3]$ performed $(\mathrm{e}, 2 \mathrm{e})$ measurements on the inner shells of a number of heavy target atoms for a primary electron beam energy of $300 \mathrm{keV}$. These measurements were performed in a transmission mode by impinging a primary electron beam onto a thin film metal target and measuring the time correlation between the electron pairs emerging from the other side. The measurements were performed on the $K$ shells of copper, silver, and gold and the $L$ shell of uranium. In the case of $K$-shell ionization, an unambiguous signature for the spin-orbit interaction of the continuum electrons in the Coulomb field of the atomic nucleus was found through the observation of strong spin asymmetries in the $(\mathrm{e}, 2 \mathrm{e})$ cross sections. For the case of the $L$-shell ionization measurements on uranium [10], where the instrumental energy resolution was sufficient to resolve the $2 p P_{3 / 2}$ from the $2 p P_{1 / 2}$ ion state, they observed spin-up/spin-down asymmetries in the angular distribution of the scattered electrons. The size of the observed spin asymmetries was too large to be accounted for by spin-orbit interaction alone and was attributed to something of the nature of a "fine structure effect" observed at low impact energies [11]. In all of these previous high-energy studies, the experimental results were compared to relativistic distorted-wave Born approximation calculations and quite good agreement between the theory and experiment was achieved.

For electron-atom ionization experiments performed at intermediate impact energies on heavy target atoms, previous work on xenon $[12,13]$ indicates that a nonrelativistic scattering theory is sufficient to describe the process, at least for the case of valence shell ionization, although the lack of a good agreement with the experiment under certain conditions still leaves this an open question [14]. Experiments were performed by the ANU group [15-17] and the group of Hanne $[2,18]$ on the ionization of the $5 p^{6}$ valence shell of xenon leading to energy-resolved $5 p^{5}{ }^{2} P_{1 / 2}$ and ${ }^{2} P_{3 / 2}$ final ion states and strong spin asymmetries were observed. A comparison of the experimental results with the calculation showed the origins of the deduced spin-asymmetries (deduced from the differences in count rate for ionization by spin-up and spin-down electrons, respectively) could to some extent be described by the fine-structure effect model $[11,19]$. This model predicts that if, in an $(\mathrm{e}, 2 \mathrm{e})$ experiment involving an atomic target, the fine structure levels of the residual ion $(l \neq 0)$ are resolved, then the ionization cross section will depend on the polarization state of the primary electron beam. It predicts nonzero spin asymmetries for collisions leading to fine-structure-resolved ion states, even in the absence of explicit spin-dependent forces acting on the continuum electrons in the initial and final states and predicts that the spin asymmetries disappear when contributions from the individual fine structure transitions are summed.

Consistent with predictions of the fine-structure effect model, distorted-wave Born approximation (DWBA) calculations $[16,2,20]$ showed that the experimental results could be largely explained by the combined effects of the electron impact-induced orientation of the ion and interference between the amplitude $f$ describing direct scattering and the amplitude $g$ describing exchange scattering [orientation alone cannot do it, asymmetry is zero if $g=0]$. However, quantitatively, significant discrepancies between the theory and the experiment were evident under certain kinematical conditions. This raised questions as to whether a more sophisticated nonrelativistic theoretical description would provide better agreement, or whether a fully relativistic scattering theory might be required to accurately describe the valence ionization of such a heavy atomic target as is the case for inner shell ionization [21]. One possible important deficiency of the DWBA lies in the fact that the final state electron-electron interaction [normally called post collision interaction (PCI)] is contained only to a first order. Brauner et al. [22] showed that PCI was very important for the intermediate energy ionization of hydrogen and that a first order treatment is inadequate. The present work is an attempt to better answer these questions and provide an extensive data set to test the accuracy of state-of the art scattering calculations. New measurements of greatly improved statistical accuracy have been performed by employing a high-sensitivity toroidal spectrometer [23] enabling simultaneous measurements to be performed over a range of electron scattering angles. The experimental results are compared to distortedwave Born approximation (DWBA) calculations and to the three-body distorted wave (3DW) calculations that contain the effects of PCI to all orders of perturbation theory.

Xenon is a particularly attractive target for experimental study for a number of reasons. Firstly, its large atomic number leads to a sufficiently large separation $(1.3 \mathrm{eV})$ between its ${ }^{2} P_{1 / 2}$ and ${ }^{2} P_{3 / 2}$ fine-structure levels to be easily resolved experimentally. Secondly, being a noble gas, it is much easier to handle experimentally than the vapors of metal atoms.

\section{EXPERIMENT}

\section{A. Background}

Traditionally, coincidence experiments have been notoriously slow due to the small capture range of momentum phase space of the electron analyzers employed. Recently such problems have been circumvented through the development of highly efficient spectrometers such as COLTRIMS $[24,25]$ and through the increasingly widespread use of multiparameter data collection techniques. The problem of extracting spin-asymmetry data from $(e, 2 e)$ data is also exacerbated by the fact that beams of polarized electrons employed for such measurements are never $100 \%$ polarized, but typically have degrees of polarization between 25 and $80 \%$, depending upon the type of cathode employed. Although contributions from the unpolarized component of the beam can be isolated, their presence leads to additional statistical errors that can mask weak spin-dependent signatures. The present 


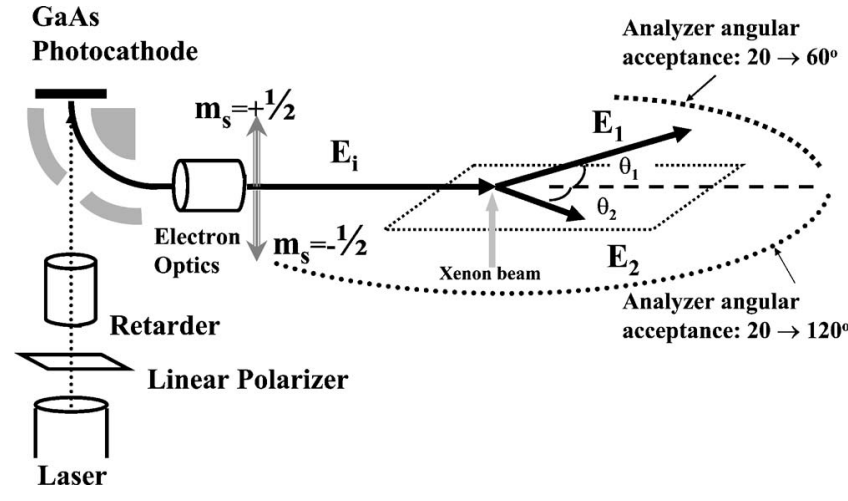

FIG. 1. Schematic representation of experimental apparatus comprising a polarized electron source, two toroidal-sector electrostatic electron analyzers (not shown) incorporating fast positionand-time-sensitive delay-line detectors.

measurements employ a source with a higher degree of polarization than those previously employed in $(\mathrm{e}, 2 \mathrm{e})$ studies on xenon and a high-efficiency toroidal spectrometer to significantly improve the statistical quality of the data.

\section{B. Apparatus}

Figure 1 shows a schematic representation of the experimental apparatus. As the apparatus has been described in previous studies $[23,26]$ only a short description will be given here. Longitudinally polarized electrons are created by the photoexcitation and extraction of valence electrons from a strained gallium arsenide photocathode under illumination by circularly polarized laser light. The structure of the photocathode is similar to those described in Nakanishi et al. [27], where values of up to $80 \%$ electron spin polarization are achieved. However, in the present case we obtain a degree of polarization of only $50 \pm 3 \%$ spin polarization for our crystal at a laser wavelength of $850 \mathrm{~nm}$. Nevertheless, this value still represents a considerable improvement over the $24 \%$ reported for our earlier xenon fine structure measurements [15].

After generation, the spin-polarized photoelectrons are extracted, deflected through $90^{\circ}$ and focused to produce a beam of transversely polarized electrons. Transverse polarization is required as only transverse components of beam polarization can lead to nonzero spin effects under the present so-called coplanar reaction geometry (Fig. 1) in which the momentum vectors corresponding to the incident and detected final-state continuum electrons are confined to a common plane, the so-called scattering plane [28]. Inversion of the electron beam polarization from into (spin down) to out of (spin up) the scattering plane is achieved by reversing the helicity of the laser light incident upon the photocathode by means of a liquid crystal retarder (Meadowlark model LVR 100). After $90^{\circ}$ deflection, the electron beam is accelerated to around $1 \mathrm{keV}$ of energy and transported through a differential pumping stage before entering the main collision chamber in which the electron spectrometer is housed. Inside this scattering chamber, the electron beam is focused and decelerated to the experimental collision energy $E_{i}$ by means of a five element electrostatic lens. The grounded interaction volume is defined by the overlap of electron and xenon beams. The required beam energy $E_{i}=\mathrm{eV}_{c}$ is set by adjusting the photocathode potential $V_{c}$. The xenon target beam is formed by effusion through a $1 \mathrm{~cm}$ long tube of $1 \mathrm{~mm}$ internal diameter oriented orthogonally to the scattering plane and collinear with the cylindrical symmetry axis of the two toroidal-sector analyzers.

Scattered electrons, emitted within the scattering plane, are momentum analyzed in one of two toroidal-sector electrostatic energy analyzers located on opposite sides of the primary electron beam. With the presently employed detection electronics (single-hit time-to-digital converters), only ionization events are measured for which the members of each final-state (e,2e) electron pair are detected in different analyzers. One analyzer transmits electrons over the polar angular range $\theta_{1}=20^{\circ} \rightarrow 60^{\circ}$, the second the range $\theta_{2}=20^{\circ}$ $\rightarrow 120^{\circ}$, where $\theta_{1}$ and $\theta_{2}$ are measured in the scattering plane with respect to the direction of the primary electron beam. However, due to the limited size of the electron detectors (80 mm diameter), parallel measurements can only be performed over a $40^{\circ}$ angular range in each analyzer. For the smaller analyzer located on the left of the incident beam, the detector is fixed at the mean angular position of $40^{\circ}$, allowing simultaneous measurement over the range $20^{\circ} \leqslant \theta_{1}$ $\leqslant 60^{\circ}$. In contrast, the detector for the larger analyzer, located on the right-hand side of the primary electron beam, is rotatable about the atomic beam axis. This enables a simultaneous measurement to be performed over an arbitrarily selected $40^{\circ}$ angular range within the angular limits $20^{\circ} \leqslant \theta_{2}$ $\leqslant 120^{\circ}$. For the present experiment, measurements were performed at mean angular positions of $40^{\circ}, 70^{\circ}$, and $100^{\circ}$ to span the full accessible $100^{\circ}$ range of slow scattered electron angles. Each analyzer comprises a seven element electrostatic lens system, four toroidal sector electrodes, and a position-and-time-sensitive electron detector. The purpose of the lens system is to select only those electrons emitted within a selected range of azimuthal emission angles $\Delta \alpha$ $\left( \pm 2^{\circ}\right.$ for this experiment), decelerate (or accelerate), and then focus them at the entrance plane of the first toroidal-sector electrode pair. As a result of the potential difference applied across the two electrode pairs, electrons are deflected $270^{\circ}$ as they pass between the electrodes and are dispersed in space according to the magnitude and direction of their initial momenta as they left the interaction region. As a result, the polar emission angle coordinates $\left(E_{i}, \theta_{i}\right)$ for a detected electron are preserved in unique arrival coordinates $\left(x_{i}, y_{i}\right)$ at the detector. Potentials applied to the toroidal-sector electrodes are chosen to determine their respective energy passbands, defined as the range of electron energies (measured at the interaction region) over which the measurement is simultaneously performed. In the present case, the passband for analyzer 1 was adjusted to span the energy interval $97.3 \mathrm{eV}$ $<E_{1}<102.7 \mathrm{eV}$ and for analyzer 2 it was adjusted to $32.3 \mathrm{eV}<E_{2}<37.66 \mathrm{eV}$. Electron energies are determined to within a small fraction of the passband energy width by the detector (400 meV for the present settings).

The detector comprises a microchannel plate electron multiplier pair followed by a crossed delay line detector from which the spatial and temporal arrival coordinates $\left(x_{i}, y_{i}, t_{i}\right)$ 
for each detected electron are measured [29]. This is achieved in the following manner. Signals from the four corners of the delay line detectors, as well as noise collected on the respective reference wires, are measured in four differential amplifier/constant-fraction discriminator units (RoentDek model DLATR6). These units discriminate against electrical noise and produce fast nuclear-instrumentmodules (NIM) output pulses, time correlated to the input pulses presented to them. The temporal arrival coordinates $t$ of the electron instigating each cascade are deduced from a fast pulse derived from the back of the second microchannel plate in each pair. This pulse is first amplified (ORTEC model AN 302/NL), and then measured in a constant-fraction discriminator (ORTEC model 934) that produces a fast NIM output pulse, time correlated to its input signal.

For each analyzer, the five fast NIM pulses associated with each measured $(\mathrm{e}, 2 \mathrm{e})$ event (one for time and four for position determination), are time delayed through $40 \mathrm{~m}$ of low-loss cable before arriving at a 16-channel 12-bit time-todigital-converter (TDC) (Phillips model 7186). This unit determines the arrival time of pulses in each channel relative to a common time reference. The time reference is provided by a fast coincidence unit (Canberra model 2144A) that produces an output pulse only when time overlap occurs between pulses from two discriminators used to process signals from the back of each microchannel plate pair.

Controlled through a CAMAC crate and a $600 \mathrm{MHz}$ laboratory computer, the TDC produces ten 12-bit numbers that contain all of the information required to deduce the $x-y$ arrival positions and arrival times for electrons at the two detectors. A separate TDC channel is used to tag events corresponding to the polarization state of the primary beam. Background events corresponding to pairs of electrons derived from separate ionization events, but nevertheless falling within the instrumental timing resolution, are subtracted using standard statistical techniques [1]. In this way, the initial momenta $\left(\mathbf{p}_{1}, \mathbf{p}_{2}\right)$ of electrons emitted in the scattering plane and comprising each measured $(\mathrm{e}, 2 \mathrm{e})$ event can be deduced.

\section{Data collection and evaluation}

The present experiment consists of measuring (e,2e) spin asymmetries for the ionization of ground state xenon atoms leading to the spin-orbit split $\mathrm{Xe}^{+} 5 p^{5}{ }^{2} P_{1 / 2}$ or $5 p^{5}{ }^{2} P_{3 / 2}$ final ion states. The reaction kinematics (shown in Fig. 2) were chosen to encompass the kinematical region of our earlier measurements $[15,16]$, where significant discrepancies between theory and experiment were found, in the hope of narrowing the previous disparities. The energy $E_{i}$ for the primary electrons is $147.8 \mathrm{eV}$. Due to the difference in binding energies of the $P_{1 / 2}$ and $P_{3 / 2}$ states $(13.44 \mathrm{eV}$ and $12.13 \mathrm{eV}$, respectively), energy conservation and the fixed-energy passbands of the two analyzers, the following differences in reaction kinematics applies between data for the two final ion states that were collected simultaneously; for the ${ }^{2} P_{3 / 2}$ state an energy integral is performed over all (e,2e) events where $98.0 \mathrm{eV}<E_{1}<102.7 \mathrm{eV}$ and $32.99 \mathrm{eV}<E_{2}<37.66 \mathrm{eV}$. For the ${ }^{2} P_{1 / 2}$ state, the energy integral is over all (e,2e) events

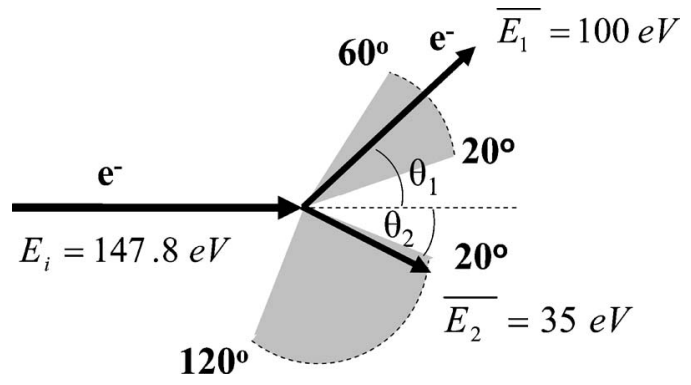

FIG. 2. Reaction kinematics. Single ionization is induced by the impact of a $147.8 \mathrm{eV}$ electron, derived from a beam of spinpolarized electrons. Fast scattered electrons of average energy $\overline{E_{1}}$ $=100 \mathrm{eV}$ are detected to the left of the primary beam, while slow scattered electrons of average energy $\bar{E}_{2}=35 \mathrm{eV}$ are detected to the right, with incident, fast and slow scattered electrons confined to a common plane (the scattering plane).

where $97.3 \mathrm{eV}<E_{1}<102.0 \mathrm{eV}$ and $32.3 \mathrm{eV}<E_{2}<37.0 \mathrm{eV}$. As a distinction from the previous data, however, the present data results from simultaneous measurement over a considerably broader range of scattering angles for the two final state electrons meaning large areas of momentum phase space are recorded under identical experimental conditions. The present higher degree of electron beam polarization has also significantly reduced the time scale of measurement, enabling the deleterious effects of time dependent changes in experimental conditions to be reduced.

The first task in the data analysis was to use Eq. (2) to sort (e,2e) events according to the final ion state $J=1 / 2$ or $J$ $=3 / 2$ with which they are associated. After sorting, spin asymmetries corresponding to transitions leading to the respective two ions states were calculated. For a final angular momentum state $J_{i}$, the spin-up spin-down asymmetry is defined as follows:

$$
A_{J_{i}}=\frac{\sigma_{J_{i}}^{\uparrow}-\sigma_{J_{i}}^{\downarrow}}{\sigma_{J_{i}}^{\uparrow}+\sigma_{J_{i}}{ }^{\downarrow}} .
$$

Here $\sigma_{J_{i}}^{\uparrow(\downarrow)}$ is the triple differential (e,2e) cross section for ionizing collisions leading to the angular momentum state $J_{i}$. The arrow is used to represent the sign of the projection of the beam polarization along the normal to the scattering plane ( $\uparrow$ for positive and $\downarrow$ for negative). Since Eq. (4) involves cross sections that are all related to the measured count rates for the spin and angular momentum resolved $(\mathrm{e}, 2 \mathrm{e})$ processes by the same proportionality constant, asymmetries can be deduced experimentally through the expression

$$
A_{J_{i}}=\frac{1}{P_{y}} \frac{N_{J_{i}}^{\uparrow}-N_{J_{i}}^{\downarrow}}{N_{J_{i}}^{\uparrow}+N_{J_{i}}^{\downarrow}},
$$

where $N_{J_{i}}^{\uparrow}$ and $N_{J_{i}} \downarrow$ are, respectively count rates for positive and negative incident-beam polarization projections for events leading to the final ion state $J_{i} ; i=1 / 2,3 / 2$ and $P_{y}$ is the component of the primary-beam spin polarization measured along the normal to the scattering plane $N_{J_{i}}^{\uparrow}$ and $N_{J_{i}}^{\downarrow}$ 
are simply determined by summing the counts under the corresponding energetically separated peaks in the binding energy spectrum after background subtraction.

\section{Energy, angular, and polarization resolution}

To improve statistics, the presented asymmetry data has been energy and angle averaged in software. The energy resolution for each of the analyzers was around $400 \mathrm{meV}$ and the energy spread of the primary beam around $300 \mathrm{meV}$, leading to an (e,2e) energy resolution of around $0.65 \mathrm{eV}$ full width at half maximum (FWHM). This was sufficient to accurately sort events according to their associated final ion states $J=1 / 2$ or $J=3 / 2$. An energy average was then performed over all measured (e,2e) energy combinations of $E_{1}$ and $E_{2}$ for transitions leading to the $J=1 / 2$ or $J=3 / 2$ ion states, corresponding to the energy bounds given in Sec. II C.

An angular average of the (e,2e) data has also been performed in software over all angular combinations corresponding to each of the two final-state continuum electrons emitted within $\mathrm{a} \pm 1^{\circ}$ range within the scattering plane (i.e., data has been sorted into $2^{\circ}$ bins). In combination with the intrinsic in-plane angular resolution of the toroidal analyzers themselves (around $\pm 1^{\circ}$ for the present lens settings), this translates into an effective angular resolution of $\pm 1.4^{\circ}$ within the scattering plane for each of the two electrons comprising each measured $(\mathrm{e}, 2 \mathrm{e})$ electron pair. Out of the scattering plane, the two toroidal analyzers accept counts over a $\pm 2^{\circ}$ range, the individual contributions to which cannot be separated in this spectrometer design.

The component of polarization $P_{y}$ was estimated by measuring the up-down spin asymmetry in the elastic scattering of electrons from xenon at $50 \mathrm{eV}$. Measured angle-resolved asymmetries were normalized to the Sherman function data of Muller and Kessler [30]. From this procedure, we estimated $P_{y}$ to be $52 \% \pm 3 \%$. According to Eq. (5), errors in measurement of $P_{y}$ translate into a systematic scaling uncertainty in the magnitude of the asymmetries and their associated errors of around $\pm 3 \%$. The errors presented on the data points of this paper's figures do not include this uncertainty, but only represent the statistical errors that vary from point to point due to variations in the counting statistics. They were calculated using standard first-order mathematical and statistical techniques, involving more complicated algebraic expressions that will not be repeated here.

\section{THEORY}

Preceding the first spin-resolved (e,2e) measurements on the ionization of heavy rare gas atoms, Jones et al. [19] first set forth a theoretical framework to investigate whether the same mechanisms that produced spin up-down asymmetries for the electron-impact excitation of the bound states of rare gas atoms with an $n p^{6}$ configuration in the ground state, would be important for the electron impact ionization studies of the rare gas atoms [31]. Their analysis was performed under the $L S$ coupling scheme with Hartree-Fock wave functions describing both the initial atomic state and final ionic states. Distorted-wave Born approximation (DWBA) calculations were carried out employing the Furness-McCarthy (FM) exchange potential [32] in the calculation of the distorted waves. The calculations predicted strong spin asymmetries for the low-energy ionization of xenon atoms within their nonrelativistic scattering framework where explicit spin-dependent forces are excluded. Within that model calculation, the spin asymmetries arose from the combined effects of an electron-impact-induced orientation of the residual ion and exchange between the incident and ejected target electron, in analogy to the previously established mechanism for spin asymmetries observed in the excitation [31]. That relativistic effects were not included in the model and might induce additional asymmetries was pointed out in their work.

The first experimental verification of the predicted spin dependence in the measured (e,2e) cross sections of xenon target atoms followed shortly after, enabling a quantitative comparison between the theoretical and experiment predictions to be made and the origin of the measured effect to be investigated. Experiments were performed at incident beam energies between 40 and $200 \mathrm{eV}$. The results were analyzed by DWBA calculations (nonrelativistic, semirelativistic, and relativistic) of varying complexity to attempt to ascertain the role and relative importance, of exchange and relativistic effects in determining the spin dependence of the observed (e,2e) cross sections.

Experimental data measured at an incident beam energy of $147 \mathrm{eV}$ by the ANU group were compared to calculations in a number of previous publications [33,34,13,15-17,20]. Both nonrelativistic and semirelativistic DWBA calculations were performed, respectively employing Hartree-Fock wave functions or Dirac-Fock wave functions to describe the target and ion wave functions. In the case of the nonrelativistic calculations, the $1.3 \mathrm{eV}$ fine-structure splitting of the ionization cross sections was allowed for by adjusting the energy of the slow outgoing electron by $1.3 \mathrm{eV}$ between the calculations for the ${ }^{2} P_{1 / 2}$ or ${ }^{2} P_{3 / 2}$ transitions. Distorted waves were calculated in the static exchange potential of the ion as appropriate. In Guo et al. [15] calculations were performed with and without the inclusion of a real Pauli potential in the calculation of the distorted waves, allowing for the possibility of spin-flip processes in the entrance and the exit channel for the continuum electrons. In Madison et al. [20,34] a postcollision interaction between the two final-state continuum electrons was included in the form of effective charges. In all cases, the semirelativistic calculations provided a significantly better description of the angular-dependent branching ratio describing the transitions leading to the respective $5 p^{5}{ }^{2} P_{1 / 2}$ or $5 p^{5}{ }^{2} P_{3 / 2}$ final ion states. Madison et al. [34] showed that the kinematical effects of including the $1.3 \mathrm{eV}$ nondegeneracy between the two fine-structure states (degeneracy is assumed for a pure fine-structure effect) had a much smaller effect than the choice of a correct description for the target wave function. In contrast to the sensitivity of the branching ratio on the target description, the asymmetry function was shown to be largely insensitive to the details of its description.

New spin-resolved measurements on xenon from the ANU group [16] and the Hanne group [2] appeared in 1998 
involving measurements at impact energies of 40,60, 100, and $200 \mathrm{eV}$ for both symmetric and asymmetric energy sharing between the two final-state continuum electrons. Theoretical efforts to explain the persistent discrepancy between the calculated and measured spin asymmetries were then intensified, to try to identify the missing physics. DWBA calculations presented in those two papers and the two theoretical papers $[12,13]$ then identified that exchange, not just between the incident and ejected target electron, but also between the continuum electrons and the electrons in the residual ion played a crucial role in determining the form of the asymmetry function. This effect was referred to as an "exchange distortion" since it modifies the differential equation for the continuum electrons and "distorts" the continuum electron wave functions. Expressions describing the relationship between the spin of the incident, scattered, and ejected electrons, and the orientation of the residual ion were also developed in Mazevet et al. [13] and calculations were performed under the ANU kinematics [16]. These calculations predicted that the exchange distortion should be the dominant spin-dependent interaction for the behavior of the asymmetry function for values of the slow electron angle less than $25^{\circ}$ and between $100^{\circ}$ and $150^{\circ}$ for those measurements.

Further development [35] of the semirelativistic DWBA approach was made in which the influence of relativistic interactions between the bound and continuum electrons was investigated. This was achieved by comparing nonrelativistic and semirelativistic DWBA calculations and assessing the sensitivity of calculated cross sections and spin asymmetries on the target description and the inclusion or omission of a spin-orbit term in the description of the continuum electrons. For the $150 \mathrm{eV}$ impact energy considered and for coplanar asymmetric kinematics, the calculations indicated that the spin-orbit interaction for the continuum electrons plays a minor role. This conclusion was further supported by the relativistic DWBA calculations of Lechner et al. [36] who used a sophisticated density functional treatment of exchange to analyze the spin asymmetries rather than less sophisticated Furness-McCarthy local potential used in earlier calculations. For the kinematics corresponding to the Münster and Canberra experiments, they concluded that the effects observed in Dorn et al. [16], which were proposed as possible evidence for continuum relativistic effects, were in fact exclusively the result of the choice used for the exchange potential. More recently [37], measurements and calculations for the double differential cross section for the low-energy ionization of xenon by spin polarized electrons were also performed. Whilst residual spin-asymmetries were observed for cross sections in which the final ion $J$ state was not resolved, the authors also expressed the opinion that this was not a strong indication of relativistic effects.

Consequently, we have a situation where there is significant disagreement between the experiment and the theory and the evidence indicates that this disagreement is not caused by relativistic effects. The important question then concerns the source of this disagreement. There are (at least) two possibilities. The first one is the importance of the final state interaction between the two continuum electrons [normally called the post collision interaction (PCI)]. Brauner,
Briggs, and Klar [22] showed that a proper treatment of PCI was very important for the electron ionization of atomic hydrogen. The DWBA approaches that have been used include PCI only to first order in perturbation theory and this may be inadequate. The second possible cause for the disagreement between experiment and theory lies in the treatment of the exchange distortion [38]. Except for the work of Lechner et al. [36], the local exchange potential of Furness-McCarthy (FM) has been used to approximate the effects of exchange distortion. Although including the FM potential consistently improves agreement between experiment and theory for xenon, perhaps an improved treatment is needed. In this paper, we will use the FM potential and investigate the importance of PCI.

Here we will present only a brief outline of the theory. More details can be found in Prideaux and Madison [39]. To examine the importance of PCI, we use the three-body distorted wave (3DW) approach. The 3DW $T$-matrix is given by

$$
T_{f i}^{3 D W}=\left\langle\chi_{f} \chi_{\text {eject }} C_{\text {proj-eject }}|V-U| \psi_{\text {active }} \chi_{i}\right\rangle .
$$

Here $\psi_{\text {active }}$ is the initial bound-state wave function for the active electron, $\chi_{i}\left(\chi_{f}\right)$ is the initial (final) state distorted wave for the projectile electron, $\chi_{\text {eject }}$ is the final state distorted wave for the ejected electron, $C_{\text {proj-eject }}$ is the Coulomb interaction between the projectile and elected electron, $V$ is the initial state interaction between the projectile and neutral atom, and $U$ is a spherically symmetric approximation for $V$.

In the 3DW T-matrix of Eq. (6), the final state Coulomb interaction between the projectile and ejected electron $C_{\text {proj-eject }}$, is included in the approximation for the final-state wave function. The important point to note is that any physics included directly in the wave function is included to all orders of perturbation theory so the 3DW has PCI included to all orders of perturbation theory. In contrast, the standard distorted-wave (DWBA) approximation does not include this interaction in the final-state wave function, as evident from its corresponding $T$-matrix element

$$
T_{f i}^{D W B A}=\left\langle\chi_{f} \chi_{\text {eject }}|V-U| \psi_{\text {active }} \chi_{i}\right\rangle .
$$

Here the presence of the PCI effects are restricted to the perturbation $(V-U)$, that means that PCI effects are included only to a first order in perturbation theory.

The initial state distorted wave is a solution of the Schrödinger equation

$$
\left(T_{\text {proj }}+U-k_{i}^{2}\right) \chi_{i}=0 .
$$

Here $T_{p r o j}$ is the kinetic energy operator for the projectile and $k_{i}^{2}$ is the energy of the incident electron. The distorting potential $U$ is given by

$$
U=U_{i}+U_{e x},
$$

where $U_{i}$ is the Hartree-Fock potential for the neutral atom and $U_{e x}$ is the FM local approximation for the exchange. We use the triplet form of the FM [32] approximation for $U_{e x}$ where the charge density is half the full density for the atom (since the projectile can only exchange with an atomic electron of identical spin). This procedure gave good agreement with the experiment for the $p$-shell ionization of xenon 


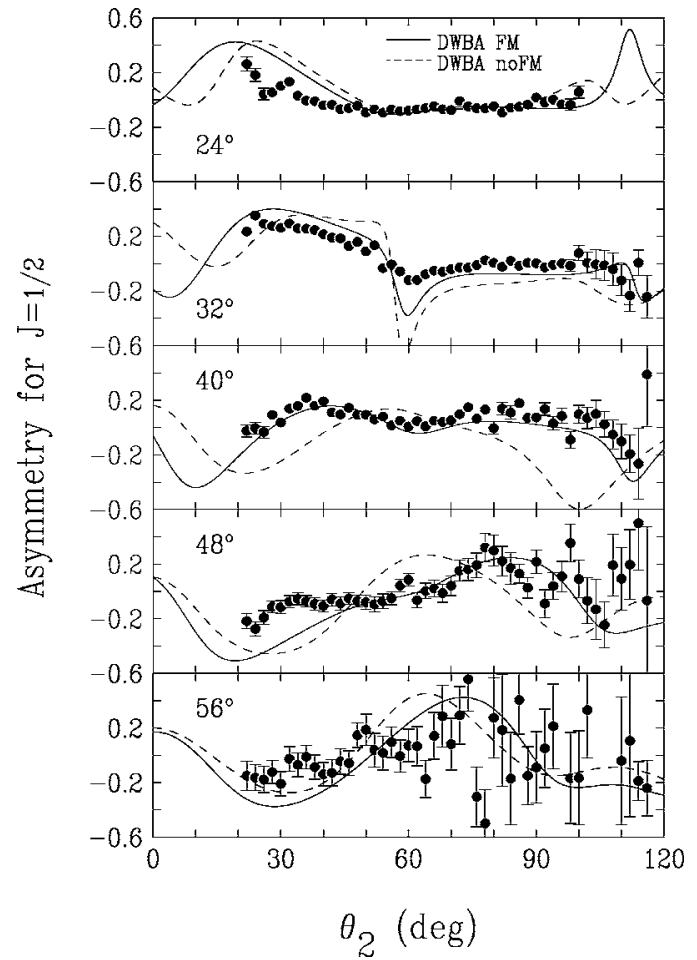

FIG. 3. Asymmetry data for ionizing transitions leading to the $\mathrm{Xe}^{+} 5 p^{5}{ }^{2} P_{1 / 2}$ state. The experimental results are compared with DWBA calculations, respectively including and excluding the Furness-McCarthy exchange potential. The fixed value of scattering angle $\theta_{1}$ is indicated in the left hand corner of each panel.

(Madison et al. [12], Mette et al. [2]). On the other hand, it should be noted that the accuracy of the local approximations for exchange distortion for $p$ shells has been questioned (Biava et al. [40]). Both final-state distorted waves are solutions of Schrödinger equations similar to Eq. (8) except that the Hartree-Fock atomic neutral potential $U_{i}$ is replaced with the Hartree-Fock potential for the final-state ion $U_{i o n}$. The final-state distorted waves are orthogonalized to $\psi_{\text {active }}$ using the Gram-Schmidt procedure. Finally, as described by Prideaux and Madison [39], if the ionization event is treated as a three-body problem, the perturbation can be approximated as follows:

$$
V-U=-\frac{1}{r_{a b}}-U_{a c t i v e},
$$

where $r_{a b}$ is the distance between the two electrons and $U_{\text {active }}$ is the spherically symmetric potential for the electronelectron interaction.

\section{COMPARISON OF THEORY WITH EXPERIMENT}

As mentioned above, it is now well established that exchange distortion (i.e., exchange between the continuum electrons and passive atomic electrons) plays an important role in spin-dependent collisions. To properly include exchange distortion, the homogeneous differential Eq. (6) would be replaced by an inhomogeneous integro-differential

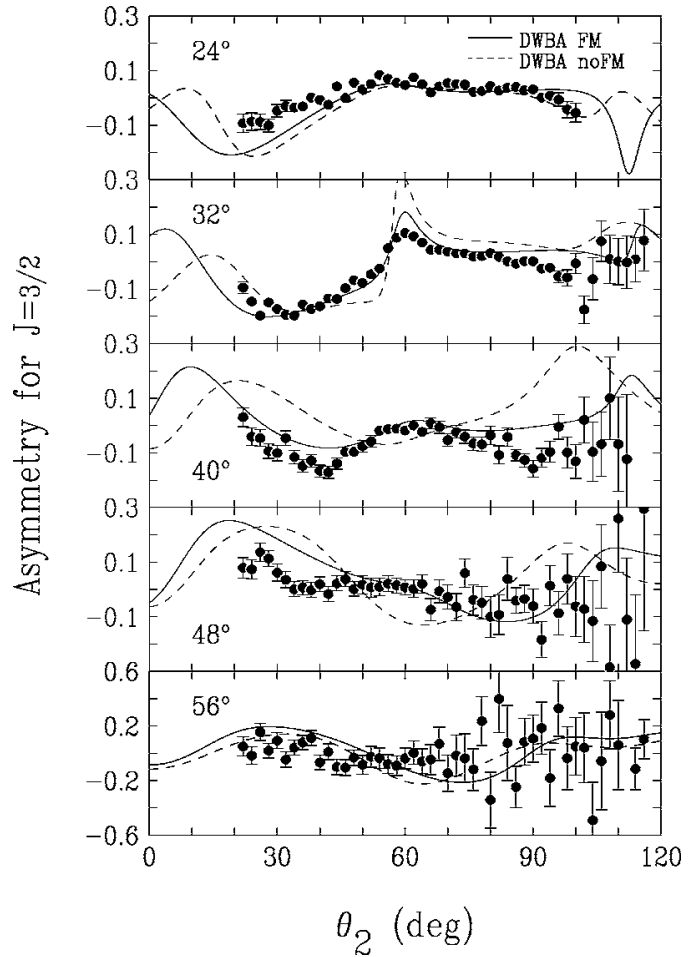

FIG. 4. Same as Fig. 3, except that the transition is to the $\mathrm{Xe}^{+}$ $5 p^{5}{ }^{2} P_{3 / 2}$ ion state.

equation with a nonlocal exchange term. In the past, the nonlocal exchange term has been approximated by the elementary local approximation of Furness and McCarthy [32] and this approximation has consistently yielded improved agreement between experiment and theory. One of the puzzling aspects of this observation lies in the fact that Biava $e t$ al. [40] investigated the accuracy of the FM potential for the electron-impact ionization of argon by comparing FM distorted with proper Hartree-Fock nonlocal potential distorted waves. The authors found that a suitable choice for the FM potential produced accurate results for the ionization of $s$ states but not $p$ states which would suggest that the FM potential should not be accurate for the present case either.

Figures 3 and 4 compare DWBA results both with and without the FM potential with the present experimental results for ionization of the $J=\frac{1}{2}$ and $J=\frac{3}{2}$ states, respectively. Statistical error bars are shown on the experimental data points where the errors are large enough to see. It is seen that, similar to earlier results, the FM potential consistently yields an improved agreement with the experiment and often the improvement is quite dramatic. Consequently, a better understanding of why the FM potential works so well awaits further detailed Hartree-Fock-type studies. In the meantime, we will use the FM potential since it clearly contains the correct type of physical effects.

Figures 5 and 6 compare DWBA and 3DW results with the present experimental results. Both calculations include the FM potential and they are identical in every respect, except that the 3DW contains the final-state Coulomb interaction $C_{\text {proj-eject }}$ in the final-state wave function such that PCI is included to all orders of perturbation theory instead of only first order. It should be noted, however, that while the 


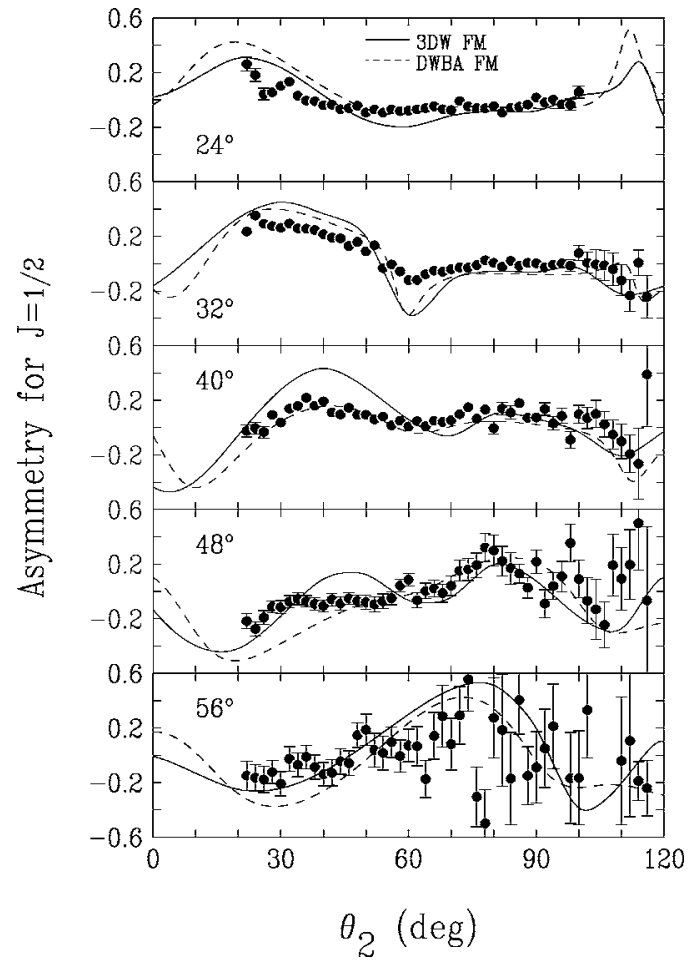

FIG. 5. Comparison of computed asymmetries from DWBA and 3DW calculations with the experimental data for ionizing transitions leading to the $\mathrm{Xe}^{+} 5 p^{5}{ }^{2} P_{1 / 2}$ state. The fixed value of scattering angle $\theta_{1}$ is indicated in the left hand corner of each panel.

DWBA runs in a few seconds on a fast PC, it takes on the order of weeks to perform the corresponding 3DW calculation on the same machine. The first thing to note from Figs. 5 and 6 is that the effect of PCI is relatively small. This is undoubtedly due to the velocity difference between the two outgoing electrons (2.7 versus 1.7 ) and the fact that there is a significant angular separation as well. In spite of the fact that there are cases for which DWBA is closer to experiment than $3 \mathrm{DW}$, overall the $3 \mathrm{DW}$ results are in better agreement with the experiment. The remaining relatively small differences between the experiment and theory might be due to inaccuracies in the FM potential.

As mentioned above, on the theoretical front we hope to be able to perform a full Hartree-Fock calculation of the distorted waves in the near future to be able to unambiguously examine the effect of exchange distortion. Experimentally, further precision measurements are desirable over a broad range of kinematical conditions in an attempt to isolate individual effects whose combined effect determine the form of the spin asymmetry functions. For example, experiments performed where both of the (e,2e) final-state continuum electrons have much higher energy with a strong asymmetric energy sharing between themselves could simplify the exchange mechanism to a largely two-body effect, enabling the influence of kinematical and target coupling effects on the asymmetries to be more accurately explored [38]. Such measurements however, will be much more challenging due to the smaller cross sections involved. Furthermore, experiments where the energy of the two final-state electrons is small $(\sim 5-10 \mathrm{eV})$ are also highly desirable. These would

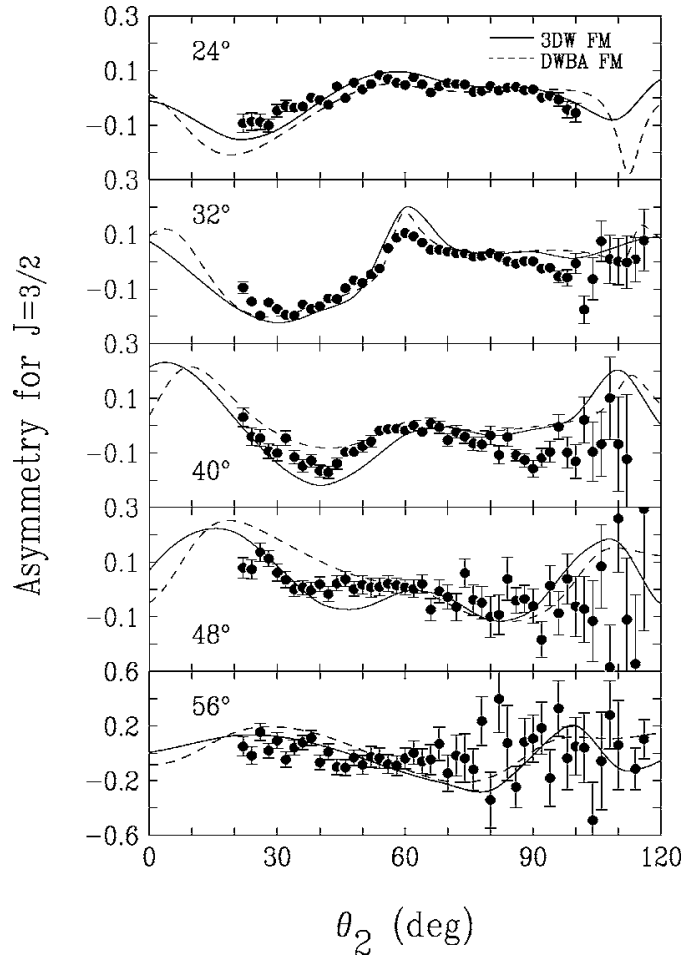

FIG. 6. Same as Fig. 5, except for transitions leading to the $\mathrm{Xe}^{+}$ $5 p^{5}{ }^{2} P_{3 / 2}$ state.

allow theory to test the applicability of the FM over an extended kinematic range; to test its strengths and limitations and gain insight into how it at least partially accounts for exchange effects under the present kinematics.

\section{CONCLUSION}

Since the first kinematically-complete spin-resolved electron-impact-induced ionization experiments some 15 years ago, dramatic advances in experimental, theoretical, and computational techniques have led to a sustained narrowing of the gap between spin asymmetries derived from measurement and theory. The spin-asymmetry function provides a highly sensitive probe to investigate exchange effects in scattering. In the absence of explicit spin-dependent forces acting on the continuum electrons, this function is identically zero in the limit of no exchange scattering. In spite of the progress, significant discrepancies between theory and experiment have persisted.

To address this issue, we presented here experimental spin-asymmetry data of much greater statistical accuracy than that previously published and high precision DWBA calculations. Overall, a good agreement between theory and experiment is achieved. For the present kinematics and adopted theoretical approach, our results show that the inclusion of PCI to all orders of perturbation does not significantly affect the result of calculation nor obviously reduce the remaining disparity between theory and experiment. In contrast, incorporation of the FM exchange potential to account for exchange distortion into our DWBA calculation 
brings significant improvement. The reasons why this elementary approximation works so well are however, not completely apparent. A more sophisticated treatment of many-body exchange effects is therefore highly desirable. To assess the degree to which kinematical and target coupling effects contribute to the remaining disparities, experiments performed over a broader kinematic range are also crucial. Together these steps promise to further enhance our understanding of the many-body nature of collision processes.

\section{ACKNOWLEDGMENTS}

The authors thank A. Dorn, H. Schmidt-Böcking, M. Smolarski, C. Höhr, A. Knapp, M. Hattas, and K. UllmannPfleger for their invaluable contributions to this work. The assistance of the Australian Research Council (ARC) under Grant No. DP0452553, the Australian-German Research Cooperation Scheme, and the U.S. NSF under Grant No. PHY0456528 is gratefully acknowledged.
[1] I. E. McCarthy and E. Weigold, in Electron-Atom Collisions, 1st ed., edited by A. Dalgarno, P. L. Knight, F. H. Read, and R. N. Zare (Cambridge University Press, Cambridge, 1995).

[2] C. Mette, T. Simon, C. Herting, G. F. Hanne, and D. H. Madison, J. Phys. B 31, 4689 (1998).

[3] M. Sauter, H. Ott, and W. Nakel, J. Phys. B 31, L967 (1998).

[4] M. Streun, G. Baum, W. Blask, J. Rasch, I. Bray, D. V. Fursa, S. Jones, D. H. Madison, H. R. J. Walters, and C. T. Whelan, J. Phys. B 31, 4401 (1998).

[5] J. Lower, E. Weigold, J. Berakdar, and S. Mazevet, Phys. Rev. Lett. 86, 624 (2001).

[6] G. Baum, W. Blask, P. Freienstein, L. Frost, S. Hesse, W. Raith, P. Rappolt, and M. Streun, Phys. Rev. Lett. 69, 3037 (1992).

[7] M. Streun, G. Baum, W. Blask, and J. Berakdar, Phys. Rev. A 59, R4109 (1999).

[8] T. Kull, W. Nakel, and C. D. Schröter, J. Phys. B 30, L815 (1997).

[9] H. T. Prinz, K. H. Besch, and W. Nakel, Phys. Rev. Lett. 74, 243 (1995).

[10] K. H. Besch, M. Sauter, and W. Nakel, Phys. Rev. A 58, R2638 (1998).

[11] J. Kessler, in Proceedings of the Fifth Summer School, Atomic and Molecular Physics and Quantum Optics, Canberra, Australia, 1992, edited by H.-A. Bachor, K. Kumar, and B. A. Robson (World Scientific, Singapore, 1992).

[12] D. H. Madison, V. D. Kratsov, and S. Mazevet, J. Phys. B 31, L17 (1998).

[13] S. Mazevet, I. E. McCarthy, and E. Weigold, Phys. Rev. A 57, 1881 (1998).

[14] A. Prideaux and D. H. Madison, J. Phys. B 37, 4423 (2004).

[15] X. Guo, J. M. Hurn, J. Lower, S. Mazevet, Y. Shen, E. Weigold, B. Granitza, and I. E. McCarthy, Phys. Rev. Lett. 76, 1228 (1996).

[16] A. Dorn, A. Elliott, X. Guo, J. Hurn, J. Lower, S. Mazevet, I. E. McCarthy, Y. Shen, and E. Weigold, J. Phys. B 30, 4097 (1997).

[17] B. Granitza, X. Guo, J. M. Hurn, J. Lower, S. Mazevet, I. E. McCarthy, Y. Shen, and E. Weigold, Aust. J. Phys. 49, 383 (1996).

[18] G. F. Hanne, Can. J. Phys. 74, 811 (1996).

[19] S. Jones, D. H. Madison, and G. F. Hanne, Phys. Rev. Lett. 72, 2554 (1994).

[20] D. H. Madison, V. D. Kravtsov, S. Jones, and R. P. McEachran, Phys. Rev. A 53, 2399 (1996).

[21] W. Nakel and C. T. Whelan, Phys. Rep. 315, 409 (1999).

[22] M. Brauner, J. S. Briggs, and H. Klar, J. Phys. B 22, 2265 (1989).
[23] J. Lower, R. Panajotovic, E. Weigold, A. Dorn, K. UllmannPfleger, C. Höhr, and O. Jagutzki, Proceedings of the International Conference, Metz 31, 2002, 172, edited by L. U. (Ancarani IOP, Bristol, 2003).

[24] R. Dörner, V. Mergel, O. Jagutzki, L. Spielberger, J. Ullrich, R. Moshammer, and H. Schmidt-Böcking, Phys. Rep. 330, 95 (2000).

[25] J. Ullrich, R. Moshammer, A. Dorn, R. Dörner, L. Ph. H. Schmidt, and H. Schmidt-Böcking, Rep. Prog. Phys. 66, 1463 (2003).

[26] J. Lower, R. Panajotovic, and E. Weigold, Phys. Scr., T 110, 216 (2004).

[27] T. Nakanishi, H. Aoyagi, H. Horinaka, Y. Kamiya, T. Kato, S. Nakamura, T. Saka, and M. Tsubata, Phys. Lett. A 158, 345 (1991).

[28] J. Kessler, in Polarized Electrons, 2nd ed., edited by G. Ecker, P. Lambropoulos, H. Walther (Springer, Berlin, 1985).

[29] ROENTDEK GMBH, www.roentdek.com

[30] H. Müller and J. Kessler, J. Phys. B 27, 5893 (1994).

[31] G. F. Hanne, in Correlations and Polarization in Electronic and Atomic Collisions and (e,2e) Reactions, Institute of Physics Conference Series, 122, edited by P. J. O. Teubner and E. Weigold (Institute of Physics, Bristol, Philadelphia, 1992), p. 15.

[32] J. B. Furness and I. E. McCarthy, J. Phys. B 6, 2280 (1973).

[33] X. Guo, J. Hurn, J. Lower, S. Mazevet, Y. Shen, I. E. McCarthy, and E. Weigold, Int. Conf. on the Physics of Electronic and Atomic Collisions, Whistler, Canada 795, 1995, AIP Conf. Proc. 360, edited by L. J. Dubé, J. B. A. Mitchell, J. W. McConkey, and C. E. Brion (American Institute of Physics, Woodbury, 1995).

[34] D. H. Madison, V. D. Kravtsov, S. Jones, and R. P. McEachran, Can. J. Phys. 74, 816 (1996).

[35] S. Mazevet, I. E. McCarthy, D. Madison, and E. Weigold, J. Phys. B 31, 2187 (1998).

[36] U. Lechner, S. Keller, E. Engel, H. J. Lüdde, and R. M. Dreizler, Electron Scattering from Atoms, Molecules, Nuclei and Bulk Matter, edited by C. T. Whelan (Kluwer/Plenum, New York, 2003), p. 131.

[37] A. Prideaux and D. H. Madison, J. Phys. B 37, 4423 (2004).

[38] M. Kampp, S. Kawano, P. J. P. Roche, J. Rasch, D. H. Madison, H. R. J. Walters, and C. T. Whelan, Eur. Phys. J. D 29, 17 (2004).

[39] A. Prideaux and D. H. Madison, Phys. Rev. A 67, 052710 (2003)

[40] A. Biava, H. P. Saha, E. Engel, R. M. Dreizler, R. P. McEachran, M. A. Haynes, B. Lohmann, C. T. Whelan, and D. H. Madison, J. Phys. B 35, 293 (2002). 